\title{
HERNIORRAFIA INGUINAL EM CRIANÇAS: valor da anestesia local associada
}

\author{
José Guilherme MINOSSI ${ }^{1,2}$, Hemerson César PICANÇO², \\ Paulo Roberto Vasconcelos PAULUCCI ${ }^{2}$, Marcelo Antônio de CARVALHO ${ }^{2,3} \mathrm{e}$ \\ Soraya VENDITES ${ }^{2}$
}

\begin{abstract}
RESUMO - Objetivo - Descrever uma técnica de anestesia local no tratamento de hérnias inguinais em crianças. Método - Foram operadas 48 crianças com hérnias inguinais sob anestesia local na Santa Casa de Misericórdia de Cerqueira César, SP, sendo 34 do sexo masculino e 14 do sexo feminino, com idades entre 3 meses e 12 anos. Apenas quatro crianças tinham hérnia bilateral. A anestesia local foi realizada com lidocaína a $1 \%$ na dose de $5 \mathrm{mg} / \mathrm{kg}$ de peso através do bloqueio dos nervos abdominogenitais próximos à espinha ilíaca ântero-superior, à altura do anel inguinal externo e na pele ao redor da incisão. A sedação foi feita com cetamina na dose de 1 a $2 \mathrm{mg} / \mathrm{kg}$ e diazepam 0,2 a $0,4 \mathrm{mg} / \mathrm{kg}$ de peso. Resultados - Todas as cirurgias puderam ser realizadas com tranqüilidade com este método, com exceção de uma criança em que o bloqueio não foi efetivo e a anestesia complementada com inalação de halogenado, sob máscara. Como complicações pós-operatórias, ocorreram três hematomas, sendo um de parede e dois em bolsa escrotal, todos com boa evolução. Conclusões - O uso da anestesia local associada à sedação é procedimento simples e seguro para realizar herniorrafias inguinais em crianças.
\end{abstract}

DESCRITORES - Hérnia inguinal, cirurgia. Anestesia local, métodos. Lidocaína. Diazepam. Criança.

\section{INTRODUÇÃO}

A anestesia local tem sido usada na prática pediátrica para prevenir dor aguda e mais comumente para aliviar a dor pós-operatória, mas sempre associada à anestesia geral ou sacral $^{(11)}$.

A justificativa para a associação de mais de um tipo de anestesia tem sido o inadequado alívio da dor com agentes analgésicos utilizados no pós-operatório e a elevada incidência de efeitos colaterais, tais como náuseas e vômitos que podem ocorrer em até $30 \%$ dos $\operatorname{casos}^{(17)}$.

A utilização de anestesia local como procedimento único não tem sido adotada pelos serviços de modo geral, embora as herniorrafias inguinais estejam sendo realizadas cada vez mais freqüentemente em regime ambulatorial ${ }^{(1,4,14,15)}$, desde os estudos pioneiros de HERZFELD ${ }^{(9)}$.

As vantagens do procedimento em regime ambulatorial têm sido realçadas por vários autores e incluem a diminuição de infecções respiratória e cruzada, diminuição dos gastos e menores alterações do comportamento ${ }^{(3,10,13)}$.

A anestesia local tem ainda como vantagem provocar distúrbios menores da função cardiorrespiratória e vascular, requerendo portanto, cuidados pós-operatórios menos intensivos. Parece diminuir a dor pós-operatória pela liberação de mediadores químicos durante sua aplicação e pode ser uma opção na falha de um bloqueio caudal ou raquidiano.

A cirurgia de correção da hérnia inguinal em crianças com anestesia local poderá ser realizada em regime ambulatorial ou com curta permanência hospitalar e sem os efeitos colaterais e riscos inerentes à anestesia geral ou caudal.

Este estudo tem o objetivo de descrever uma técnica de anestesia local utilizada em crianças para o tratamento cirúrgico de hérnias inguinais, usada como procedimento principal para se obter analgesia durante a operação. A sedação foi necessária para contenção da criança, já que a maioria não é cooperativa nesta faixa etária.

Trabalho realizado na Santa Casa de Misericórdia de Cerqueira César, SP.

${ }^{1}$ Disciplina de Gastroenterologia Cirúrgica da Faculdade de Medicina de Botucatu - Universidade Estadual Paulista, Botucatu, SP. ${ }^{2}$ Santa Casa de Misericórdia de Cerqueira César, SP. ${ }^{3}$ Santa Casa de Misericórdia de Piraju, SP.

Endereço para correspondência: Dr. José Guilherme Minossi - Praça Irmãos Ferreira, 171 - 18760-000 - Cerqueira César, SP. e-mail: jminossi@ fmb.unesp.br 


\section{MÉTODO}

No período de janeiro de 1992 a dezembro de 2000, foram realizadas 48 herniorrafias inguinais em crianças sob anestesia local e sedação na Santa Casa de Misericórdia de Cerqueira César, SP.

Das crianças operadas, 34 eram do sexo masculino e 14 do feminino. A idade variou de 3 meses a 12 anos.

A maioria foi de casos de hérnia inguinal unilateral (44) e 4 casos de hérnia bilateral, sendo que destes últimos 3 eram do sexo feminino.

As hérnias inguinais com anestesia local corresponderam a $60 \%$ das herniorrafias executadas no período (48 num total de 96 herniorrafias).

O estudo realizado foi prospectivo, tendo sido elaborada ficha para dados de identificação, tipo de procedimento, complicações intra e pós-operatórias, uso de antimicrobianos, quantidade de anestésico e de sedativos utilizados e efeito da anestesia.

Os pacientes que deram entrada no serviço com diagnóstico de hérnia inguinal foram avaliados inicialmente quanto à possibilidade de serem submetidos a intervenção com anestesia local, sendo o primeiro passo a aceitação por parte dos pais. Foram excluídas as crianças que apresentavam grandes hérnias inguinoescrotais, hérnias encarceradas ou hérnias recidivadas. Também foram excluídas da casuística crianças obesas e aquelas cujos pais não aceitaram o procedimento; hérnia inguinal bilateral foi um fator de exclusão relativo.

\section{Técnica Anestésica}

Foi utilizada a lidocaína a $1 \%$ sem vasoconstrictor, em volumes variáveis, na dose de $5 \mathrm{mg} / \mathrm{kg}$ de peso, podendo chegar, excepcionalmente, a $7 \mathrm{mg} / \mathrm{kg}$. Quando o volume de anestésico a ser injetado era inferior a $10 \mathrm{~mL}$, este foi diluído em água destilada, ao meio ou até completar $10 \mathrm{~mL}$. Quando o volume de anestésico a ser injetado era superior a $10 \mathrm{~mL}$, não se realizava diluição.

A anti-sepsia prévia à anestesia local foi realizada com álcool iodado $2 \%$ e mais recentemente, com clorexidina alcoólica a $0,5 \%$, somente na região inguinal. Em seguida à anestesia regional, procediase à anti-sepsia completa e colocação de campos estéreis. Para o bloqueio foram utilizadas seringas de 5 ou $10 \mathrm{~mL}$ e agulha $13 \times 4,5$.

A primeira injeção foi realizada através da introdução perpendicular da agulha, 1 a $2 \mathrm{~cm}$ medialmente à espinha ilíaca ântero-superior. Neste ponto, após perfurar a aponeurose do músculo oblíquo externo, foram injetados aproximadamente $40 \%$ do volume total programado, num único ponto. Em crianças maiores, o anestésico foi injetado em três pontos, sendo os outros dois laterais, após retornar a agulha até o subcutâneo e penetrar novamente a aponeurose, semelhante à técnica usada no adulto. Nesta circunstância, o volume anestésico deve ser dividido por 3 (Figura 1).

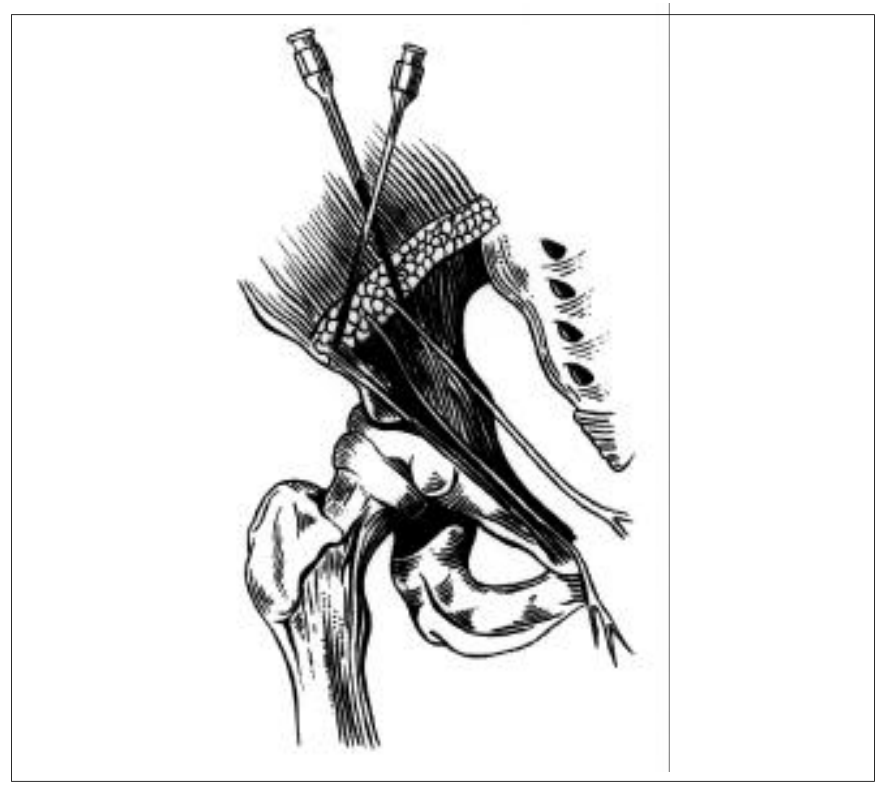

FIGURA 1 - Inervação da região inguinal e local do bloqueio dos nervos abdominogenitais

Esta técnica visa o bloqueio anestésico dos dois nervos abdominogenitais (iliohipogástrico e ilioinguinal) e deve ser considerada a mais importante para o sucesso da cirurgia.

O segundo ponto de injeção situou-se na altura do anel inguinal externo, próximo ao tubérculo púbico, onde foi feita uma injeção de aproximadamente $20 \%$ do volume anestésico, com o cuidado de não atingir os elementos do cordão, que muitas vezes têm um trajeto subcutâneo longo neste local (Figura 2).

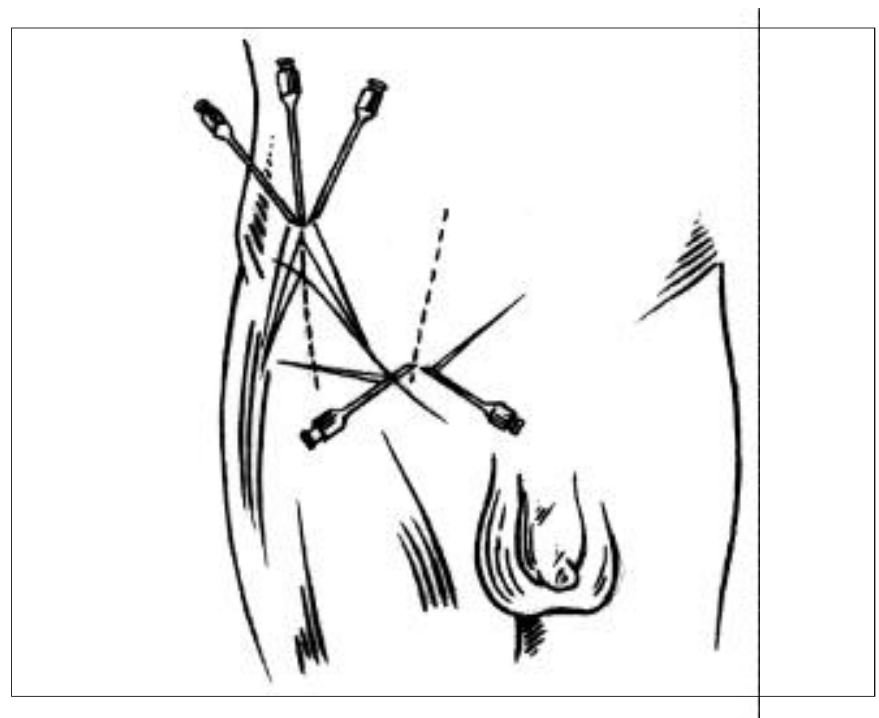

FIGURA 2 - Bloqueio anestésico dos nervos ileohipogástrico e ileoinguinal medialmente à espinha ilíaca ânterosuperior e ao nível do anel inguinal externo 
A última injeção foi feita na linha de projeção da incisão e a seu redor ou em leque, nas crianças maiores. $\mathrm{O}$ volume aqui injetado foi também de aproximadamente $20 \%$ do total. Os restantes $20 \%$ foram guardados e utilizados, se necessário, durante o procedimento cirúrgico, principalmente durante a tração do saco herniário, o que pode levar à dor, quando o primeiro bloqueio não for efetivo.

\section{Sedação}

A sedação foi sempre realizada pelo médico anestesista após monitorização, na entrada do centro cirúrgico ou na sala cirúrgica, quando se tratava de criança cooperativa. Após a sedação, todos eram monitorizados com oximetria de pulso e cardioscópio.

Para sedação foi sempre utilizada a cetamina na dose de 1 a $2 \mathrm{mg} /$ $\mathrm{kg}$, via endovenosa, juntamente com diazepam 0,2 a $0,4 \mathrm{mg} / \mathrm{kg}$, numa mesma seringa. A seringa foi preparada com $2 \mathrm{mg} / \mathrm{kg}$ de peso de cetamina e $0,4 \mathrm{mg} / \mathrm{kg}$ de diazepam, sendo administrada metade desta dose. Se a criança começava a acordar antes do término da cirurgia, doses adicionais desta mistura foram administradas.

\section{Técnica Cirúrgica}

Foi utilizada incisão transversa ou oblíqua na região inguinal, abrindo pele, tecido subcutâneo e fáscia, seguida de hemostasia de vasos sangrantes com fios de catgut simples 3-0 ou 4-0. Aberta a aponeurose do grande oblíquo, foi isolado funículo espermático no sexo masculino e ligamento redondo em crianças do sexo feminino, sendo que este eventualmente foi ligado distalmente para facilitar a dissecção do saco herniário. O saco herniário, dissecado até a altura do anel inguinal interno, foi aberto e seu conteúdo analisado. A ligadura do saco foi realizada na altura do anel inguinal através de sutura transfixante com fio trançado de poliéster 3-0. A manobra de Barker foi realizada raramente. Nenhum tipo de reforço foi realizado. A hemostasia foi revisada e a aponeurose do grande oblíquo fechada com pontos de algodão 3-0. O tecido subcutâneo foi fechado com pontos separados de catgut 3-0 simples e a pele com fio monofilamentar de nylon 4-0 ou 5-0, pontos separados.

\section{RESULTADOS}

Todas as cirurgias puderam ser realizadas com tranqüilidade com este método, com exceção de uma criança em que o bloqueio não foi efetivo, necessitando de complementação anestésica com máscara de um halogenado.

Nenhuma criança necessitou de intubação traqueal, embora 26 crianças (54\%) tivessem apresentado apnéia transitória com queda da saturação de oxigênio, necessitando de ventilação com máscara de oxigênio. Seis pacientes necessitaram de doses adicionais do anestésico e em apenas dois a dose de lidocaína foi a máxima admitida $(7 \mathrm{mg} / \mathrm{kg})$.
O tempo operatório variou de 10 a 32 minutos, sem intercorrências operatórias.

Quanto às complicações pós-operatórias, ocorreram três hematomas, sendo um de parede, no pós-operatório imediato em uma criança de 1 ano e dois casos de hematomas em bolsa escrotal em crianças de 4 e 5 meses, sendo que um caso necessitou de drenagem cirúrgica. Todos evoluíram bem.

A alta hospitalar ocorreu antes de 24 horas após a cirurgia em 47 crianças. Apenas uma permaneceu internada por 36 horas.

\section{DISCUSSÃO}

O reparo de hérnias inguinais com anestesia local em adultos é hoje procedimento bem estabelecido e seguro ${ }^{(8,12,18)}$, apresenta custos reduzidos $^{(2,16)}$ e aparentemente menor taxa de recidiva ${ }^{(2)}$; não aumenta a taxa de complicações pós-operatórias ${ }^{(16)}$, além do que a dor pósoperatória parece ser de menor intensidade devido à liberação de mediadores químicos.

Em crianças, no entanto, não tem sido descrito tratamento cirúrgico de hérnias inguinais com anestesia local exclusiva, mas esta técnica tem sido usada, como parte de uma anestesia balanceada, para melhora da dor pós-operatória e evitar os efeitos colaterais de analgésicos e narcóticos $^{(6,11)}$.

Como as crianças apresentam, de modo geral, dor de menor intensidade que os adultos e deambulam rapidamente no pósoperatório, não houve motivos para realizar dois procedimentos anestésicos para tratamento desta patologia.

Quanto à técnica anestésica utilizada, adotou-se a mesma de outros autores em pacientes adultos, principalmente as descritas por CHRISTMANN et al. ${ }^{(7)}$, com pequenas modificações, principalmente quanto ao anestésico utilizado, que parece não influir nos resultados ${ }^{(12)}$.

Embora a maioria dos autores utilize a bupivacaína na dose de 1$2 \mathrm{mg} / \mathrm{kg}$ de peso, onde volumes maiores podem ser injetados, preferiuse a lidocaína na dose de $5 \mathrm{mg} / \mathrm{kg}$ de peso. Não se tem utilizado vasoconstrictor, pois esta prática pode ter sido a causa de hematomas quando se iniciou a anestesia local em adultos submetidos a herniorrafia inguinal ${ }^{(12)}$, o que também foi sugerida por outro autor ${ }^{(8)}$.

É provável que, se forem necessárias doses maiores de lidocaína como $7 \mathrm{mg} / \mathrm{kg}$, a associação com adrenalina 1: 200.000 se faça necessária, pois o uso do vasoconstrictor nesta situação reduz a toxicidade sistêmica dos anestésicos locais por redução de irrigação sangüínea na área da injeção ${ }^{(17)}$.

Nos únicos dois casos em que se teve que usar a dose de $7 \mathrm{mg} / \mathrm{kg}$ para concluir a operação, o vasoconstrictor não foi utilizado pois já haviam sido injetados $5 \mathrm{mg} / \mathrm{kg}$ do anestésico sem o mesmo. Não foi observada toxicidade sistêmica nesses casos.

Como já mencionado, o aspecto técnico mais importante consiste no bloqueio do nervo ileohipogástrico e ileoinguinal. O nervo 
ileohipogástrico é o mais importante pois supre feixes do músculo oblíquo externo, peritônio parietal e um ramo cutâneo anterior inerva a pele e tecido subcutâneo da região inguinal, sendo este mais facilmente bloqueado. $\mathrm{O}$ nervo ileoinguinal inerva a região do anel inguinal profundo, parte superior interna da coxa e parte anterior do escroto em garotos e monte pubiano em garotas. Um volume razoável de anestésico depositado abaixo da aponeurose do oblíquo externo, medialmente à espinha ilíaca ântero-superior (aproximadamente 1,0 $\mathrm{cm}$ na criança e 2,0 cm em adultos) é capaz de bloquear ambos estes nervos.

Entretanto, um número de bloqueios falhos pode ocorrer se apenas esta técnica for usada, sendo melhor que a agulha penetre 2 ou 3 pontos medialmente à espinha ilíaca, conforme descrito, para bloquear o nervo ileohipogástrico e o nervo subcostal se ele estiver envolvido em dermátomos aferentes.

Estes bloqueios são mostrados nas Figuras 1, 2.

Cuidado que se deve ter durante o bloqueio anestésico é que o depósito em local muito próximo ao ligamento inguinal, inferiormente à espinha ilíaca e em grandes quantidades, pode resultar em bloqueio temporário do nervo femoral, com paralisia do músculo quadríceps e, conseqüentemente, retardar a deambulação do paciente.

$\mathrm{Na}$ presente casuística, o bloqueio foi efetivo em 47 pacientes, sendo que em apenas um a anestesia foi complementada por via inalatória (máscara de halotano). Em 41 pacientes a cirurgia foi realizada sem necessidade de complementar a anestesia local e em 6, doses adicionais foram necessárias.

Para sedação das crianças foi utilizada a associação de cetamina e diazepam. A cetamina é anestésico quimicamente relacionado às fenciclidinas e às ciclohexaminas que produz um estado descrito como anestesia dissociativa, sendo que o paciente se apresenta cataléptico, mas com analgesia, amnésia e sedação superficial.

Embora este anestésico promova boa analgesia na pele e no tecido subcutâneo, isoladamente não permite a execução do ato cirúrgico. Tem ainda como vantagens os mínimos efeitos nos parâmetros respiratórios e parece manter os reflexos protetores contra a regurgitação e broncoaspiração ${ }^{(5)}$. A recuperação da consciência ocorre após 10 a 15 minutos da injeção intravenosa, tempo que coincide com o período de duração de maior parte das herniorrafias. Tem como maior inconveniente a presença de delírios e sonhos desagradáveis que podem ser amenizados com o uso de benzodiazepínicos, daí a associação com diazepam.
Embora as alterações dos parâmetros respiratórios sejam raras com uso de cetamina nas doses utilizadas, certamente a associação com diazepínicos aumenta os riscos de depressão respiratória, o que ocorreu em $54 \%$ dos casos desta série, sendo portanto obrigatório o uso de monitorização cardíaca, oximetria e ter à mão todo material necessário para ressuscitação, além de que um médico anestesista deve estar sempre presente à cabeceira do paciente para que possa tratar intercorrências e mudar o ato anestésico em caso de falha do bloqueio.

Outro aspecto que merece comentário é que crianças pequenas (de menos de 1 ano) têm coxim gorduroso na região inguinal, assim como as obesas, o que pode dificultar um pouco a operação. Por outro lado, crianças maiores que 1 ano, magras, do sexo feminino e com hérnias unilaterais são os casos ideais para se realizar a herniorrafia com anestesia local. Nos casos em que a doença é bilateral, podem ser necessárias grandes doses de anestésicos e se estas ultrapassarem a dose máxima preconizada, o procedimento não deverá ser, portanto, indicado. Na presente casuística, quatro crianças apresentavam hérnia bilateral, sendo três do sexo feminino e magras. Nesses casos conseguese realizar o bloqueio bilateral e não ultrapassar a dose máxima do anestésico, principalmente porque na mulher o procedimento é muito mais simples, pois não existe o funículo espermático e a dissecção do saco herniário é mais fácil. O paciente do sexo masculino operado também era um caso favorável, por ser criança longilínea, magra e com hérnia oblíqua externa pequena.

Com relação às complicações, não ocorreu nenhuma durante a cirurgia. No pós-operatório observou-se três casos de hematoma, sendo um de parede e dois de bolsa escrotal em crianças de 4 e 5 meses. Um caso necessitou de drenagem cirúrgica e a alta ocorreu em 36 horas. Nenhum caso de infecção da ferida ou de outros locais foi observado.

É possível concluir que a anestesia local para correção de hérnias inguinais em crianças é, à semelhança do que ocorre em adultos, procedimento simples e seguro, devendo ser realizado sempre em ambiente que conte com toda monitorização para procedimentos maiores e com a presença de profissional médico, de preferência anestesiologista, para realizar a sedação e os cuidados com a mesma.

\section{AGRADECIMENTO}

Ao Prof. Dr. Elson Felix Mendes pela revisão do texto e valiosas sugestões. 
Minossi JG, Picanço HC, Paulucci PRV, Carvalho MA, Vendites S. Inguinal hernia repair in children: importance of local anesthesia association. Arq Gastroenterol 2002;39(3):204-208.

ABSTRACT - Aim - To describe an anesthetic technique, as well as the results of surgical treatment of the inguinal hernia in children. Patients/ Methods - Forty-eight patients were submitted to inguinal hernia repair under local anesthesia at "Santa Casa de Misericórdia de Cerqueira César", State of São Paulo, Brazil. There were 34 male and 14 female patients, range from 3 months to 12 years old. Local anesthesia was performed with a dose of $5 \mathrm{mg} / \mathrm{kg}$ body weight of $1 \%$ lidocaine through iliohypogastric and ilioinguinal nerve blocks, medially to the anterior superior iliac spine, and at level of the pubic tubercle. Sedation was done with an association of ketamine (1 to $2 \mathrm{mg} / \mathrm{kg})$ and diazepam ( 0,2 to $0,4 \mathrm{mg} / \mathrm{kg}$ ). Results - In all patients except one the procedure was done without complications. In that patient the local anesthesia was not effective and was followed by inhalatory anesthesia. Surgical complications (blood collections) were observed in three patients: two at the scrotum and one at subcutaneous, with good evolution. Conclusions - We conclude that the use of local anesthesia associated to sedative is a simple and safe procedure for the inguinal hernia repair in children.

HEADINGS - Hernia, inguinal, surgery. Anesthesia, local, methods. Lidocaine. Diazepam. Child.

\section{REFERÊNCIAS BIBLIOGRÁFICAS}

1. Ahlgren EW. Pediatric outpatient anesthesia. A four-year review. Am J Dis Child 1973;126:36.

2. Baskervelle PA, Jarrett PEM. Day case inguinal hernia repair under local anesthetic. Ann R Coll Surg Engl 1963,65:224.

3. Beltran-Brown F. Cirurgia pediátrica. México: Ediciones Médicas del Hospital Infantil; 1969.

4. Benson DM, Root B. An outpatient pre-anesthetic evaluation service. Ohio State Med J 1979;75:139-43.

5. Braz JRC. Anestesia venosa. In: Braz JRC, Castiglia YMM, editores. Temas de anestesiologia. 2a ed. São Paulo: Editora UNESP/Artes Médicas; 2000. p.101-3.

6. Cohen MM, Cameron CB, Duncan PG. Pediatric anesthesia morbidity and mortality in the perioperative period. Anesth Analg 1990;70:160-7.

7. Christmann EF, Raffo JM, Ottolenghi CE, Grolman GV. Técnica cirúrgica. 2a ed. bras, traduzida da 8a ed. argentina. Rio de Janeiro: Guanabara Koogan; 1958. v.2, p. 1224-5.

8. Glassow F. Inguinal hernia repair using local anaesthesia. Ann R Coll Surg Engl 1984;66:382-7

9. Herzfeld G. Hernia in infant. Am J Surg 1938;39:422

10. Izant RJ. Non-operative aspects of pediatric surgery. In: Report of the $27^{\text {th }}$ Ross Pediatric Research Conference. Columbus, Ohio; 1957.
11. McNicol LR. Local anaesthesia. In: Morton NS; Raine PAM, editors. Paediatric day case surgery. Oxford, NY: Oxford University Press; 1994. p. 24-43.

12. Minossi JG, Pedro FAJ, Vendites S. Herniorrafia inguinal com anestesia local. Arq. Gastoenterol 1992;29:18-22.

13. Othersen HB, Clatworthy HW. Outpatients herniorrhaphy for infants. Am J Dis Child 1968;116:78-80

14. Thompson GE, Remington MJ, Millman BS, Bridenbaugh LD. Experiences with outpatient anesthesia. Anesth Analg 1973;52:881-7.

15. Treloar J. Outpatient anesthetic practice in Vancouver. Anaesth Intensive Care $1973 ; 1: 250$.

16. Vater J, Gonçalves MCV. Ampliação do binômio anestesia local-paciente externo: enfoque especial para as hérnias inguinocrurais. Resid Méd (Rio de J) 1987; 15:10, 13-4, 17-22.

17. Vianna PTG, Módolo NSP. Anestésicos locais. In: Braz JRC, Castiglia YMM, editores. Temas de anestesiologia. 2 $2^{\text {a }}$ ed. São Paulo: Editora UNESP/Artes Médicas; 2000. p. 134.

18. Von Bahr V. Anestesia local na herniorrafia inguinal. In: Mello JMS, editor. Manual ilustrado de anestesia local. São Paulo: Publicações Médicas; 1987. p.30-1.

Recebido em 12/3/2002 Aprovado em 22/6/2002. 